\title{
Maximization of Information in Energy-Limited Directed Communication
}

\author{
Touraj Soleymani, Student Member, IEEE, Sandra Hirche, Senior Member, IEEE, \\ and John S. Baras, Life Fellow, IEEE
}

\begin{abstract}
This study is concerned with an observer who desires to inform optimally a distant agent regarding a physical stochastic process in the environment. We consider that the observer has a constraint on the energy resource for the directed communication to the agent. The information we are interested in is the change in the knowledge possessed by the agent about the state of the process. We find the maximum information that can be transferred from the observer to the agent over a finite horizon subject to a bound on the total energy of the observer. We show that the maximum information is the optimal value of a mixed-integer nonlinear optimization problem. We obtain lower and upper bounds on the maximum information and also a suboptimal admissible solution based on a semidefinite programming. Moreover, we propose an optimized event-triggering mechanism based on a linear matrix inequality which yields event-driven sampling. Numerical and simulation results are presented for simple stable and unstable systems.

Index Terms-Maximum Information, Nonuniform Sampling, Time-Varying Precision, Time-Varying Quantization.
\end{abstract}

\section{INTRODUCTION}

This study is concerned with an observer who desires to inform optimally a distant agent regarding a physical stochastic process in the environment. If the observer is not constrained in terms of energy, medium, security, etc., then it should transmit its measurements continually with the highest rate and quality. On the contrary, here we consider that the observer has a constraint on the energy resource for the directed communication to the agent, i.e., the observer stops operating when its energy is depleted. An example is a wireless sensor network that relies on a capacity-limited cell, and wishes to have an extended duration of operation. In this situation, the observer should transmit measurements that contain more valuable information. The information we are interested in is the change in the knowledge possessed by the agent about the state of the process. We find the maximum information that can be transferred from the observer to the agent over a finite horizon subject to a bound on the total energy of the observer. Applications of this study include environmental monitoring, planetary exploration, wearable sensing, teleoperation, and many other examples of networked systems.

In our problem, the observer transmits its measurements through a binary channel, and the decision making takes

T. Soleymani and S. Hirche are with the Institute for Information-Oriented Control, Technische Universität München, D-80333 Munich, Germany (email: $\{$ touraj, hirche\}@tum.de).

J. S. Baras is with the Institute for Systems Research, University of Maryland College Park, MD 20742, USA (email: baras @umd. edu).

T. Soleymani and J. S. Baras are also with the Institute for Advanced Study, Technische Universität München, D-85748 Garching, Germany.

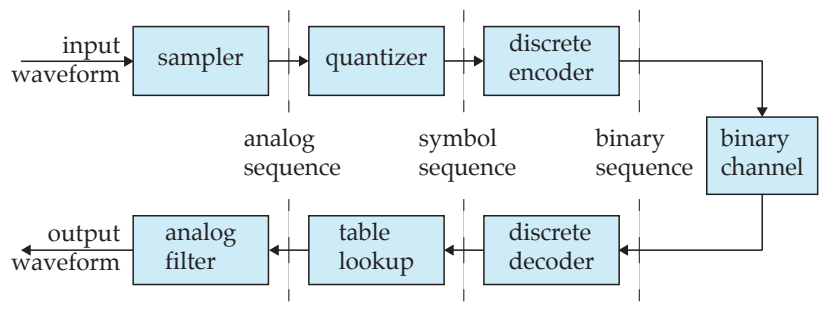

Fig. 1. Three layers of source encoding/decoding.

place inside a source encoder. Fig. 1 illustrates three layers of source coding in digital communication. We use a continuous-discrete time linear dynamical model for the system, and consider the energy resource as a hard constraint. We employ nonuniform sampling and time-varying precision and quantization to enable the observer to transmit measurements that contain more valuable information, and show that the maximum information is the optimal value of a mixedinteger nonlinear optimization problem. The main contributions of this paper are as follows: we obtain lower and upper bounds on the maximum information and also a suboptimal admissible solution based on a semi-definite programming (SDP); moreover, we propose an optimized event-triggering mechanism based on a linear matrix inequality (LMI) which yields event-driven sampling.

The outline of this paper is as follows. After a brief literature survey and an introduction on notation, the problem formulation is presented in Section II. We study the optimization problem for maximizing the information in Section III. In Section IV, we derive bounds on the information. In Section $\mathrm{V}$, we propose an event-triggering mechanism for sampling. We illustrate numerical and simulation results in Section VI. Concluding remarks are made in Section VII.

\section{A. Related Work}

There is a large and growing literature on nonuniform sampling and sensor scheduling in estimation theory. Meier et al. [1] extend the work of Kushner [2] by looking at the measurement control problem subject to measurement cost and constraints, and by proposing dynamic programming (DP) and the gradient method as computational procedures. Recently, Rabi et al. [3] study optimal sampling as a stopping time problem for a scalar system under a finite transmission budget constraint. Molin and Hirche [4] investigate the optimal design for sampling in a scalar system with a communication cost by considering a two-player problem. 
Sijs and Lazar [5] study event-driven sampling based on measurements with an asymptotic bound on the error covariance; whereas Trimpe and D'Andrea [6] propose an eventtriggering mechanism based on the error covariance, and study its asymptotic properties.

Moreover, Baras and Bensoussan [7] study sensor scheduling in nonlinear filtering of diffusion processes with switching cost. Savkin et al. [8] address the sensor scheduling problem for continuous-time linear systems, while Krishnamurthy and Evans [9] consider the problem for hidden Markov models. Later, Gupta et al. [10] propose a stochastic strategy for the sensor selection problem. Sandberg et al. [11] study sensor scheduling with communication cost for sensors with different qualities and time-delays. Wu and Arapostathis [12] look at the sensor scheduling problem over an infinite horizon for partially observable Markov decision processes, while Ny et al. [13] consider the infinite horizon problem for continuous-time linear systems.

In this paper, the system should decide on sampling times, precision modes, and quantization step-sizes while there is a constraint on its energy resource. The maximization of information problem, as we will see, is an NP-hard optimization problem. We propose an efficient SDP-based algorithm in order to obtain bounds on the maximum information. In addition, we shed light on the event-driven sampling problem by casting it as a special case of a general problem.

\section{B. Notation}

In this paper, we represent an $n$ dimensional vector with $x=\left[x_{1}, \ldots, x_{n}\right]^{T}$ where $x_{i}$ is its $i$ th component. We write $x^{T}$ to denote the transpose of the vector $x$. The identity matrix with dimension $n$ is denoted by $I_{n}$. We use $x_{1: k}$ to denote the sequence $\left\{x_{1}, \ldots, x_{k}\right\}$. We write $\delta_{t-t^{\prime}}$ and $\delta_{s s^{\prime}}$ to denote the Dirac and Kronecker delta functions, respectively. We write $p(x)$ to denote the probability distribution of the stochastic variable $x$. The normal distribution with mean $\mu$ and covariance $\sigma^{2}$ is denoted by $N\left(\mu, \sigma^{2}\right)$. For matrices $A$ and $B$, we write $A \succ 0$ and $B \succeq 0$ to mean that $A$ and $B$ are positive definite and positive semi-definite, respectively.

\section{Problem Formulation}

\section{A. Physical Process and Source Encoder Models}

We use a continuous-discrete time model [14] to describe our system. This is due to the fact that the state of the process evolves in continuous time, and that the measurements are obtained at discrete instants of time. Consider a stochastic continuous-time physical process in the environment which is generated by the following linear stochastic differential equation:

$$
\dot{x}_{t}=A_{t} x_{t}+B_{t} w_{t}
$$

where $x_{t} \in \mathbb{R}^{n}$ is the state of the system at time $t, A_{t}$ and $B_{t}$ are state and input matrices at time $t$, and $w_{t} \in$ $\mathbb{R}^{m}$ is a white noise process with zero mean and covariance $Q_{t} \delta_{t-t^{\prime}}$ where $Q_{t} \succ 0$. The initial state $x_{0}$ is assumed to be a Gaussian vector with zero mean and covariance $P_{0}$. An observer who desires to inform a distant agent regarding the state of the process employs a source encoder to transmit its measurements in the form of a sequence of bits.

In particular, first a sampler that is equipped with a sensor samples the process at time instants $t_{s}$ for $s=1,2, \ldots, M$ where $M$ can be infinite over the time horizon $[0, T]$. The measurement model of the sensor is given by

$$
y_{s}=C_{s} x_{s}+v_{s}
$$

where $y_{s} \in \mathbb{R}^{p}$ is the output of the sampler at time instant $t_{s}, C_{s}$ is the output matrix at time instant $t_{s}, x_{s}$ is the state of the process at time instant $t_{s}$, and $v_{s}$ is a white noise sequence with zero mean and covariance $\left(R+\Delta R_{s}\right) \delta_{s s^{\prime}}$ where $R$ is the base covariance of the sensor, $\Delta R_{s}$ is the mode parameter, and $R+\Delta R_{s} \succ 0$.

Remark 1: The sensor is assumed to have different precision modes. At time instant $t_{s}$, the mode of the sensor is specified by the pair $\left(C_{s}, \Delta R_{s}\right)$.

The sample $y_{s}$ is then quantized by a uniform quantizer. We model the quantizer output by

$$
z_{s}=y_{s}+n_{s}
$$

where $z_{s}$ is the quantizer output at time instant $t_{s}$ and $n_{s}$ is an uncorrelated white additive noise sequence with zero mean and covariance $\Delta q_{s}^{2} I_{p} \delta_{s s^{\prime}} / 12$ where $\Delta q_{s}$ is the quantization step-size. We use the convention $\Delta q_{0}=0$ for the ideal quantization. The validity of the additive white noise model for quantization and the related conditions are studied in [15].

Remark 2: In our setting (i.e. single observer and decision maker), sampling and quantization are mathematically commutable operations. It makes no difference whether a signal is first sampled and then the samples are quantized, or vice versa. This is not necessarily the case when multiple observers and decision makers are involved.

Finally, an encoder codes the quantized sample $z_{s}$. The bits encoding the codewords of $z_{s}$ are then transmitted to the agent through a noiseless zero-delay binary channel. Therefore, at time $t \geq t_{s}$ the set of measurements $z_{1: s}$ is available to the agent. Through this study, the agent assumes that the observer is trustable and that measurements are never compromised.

Remark 3: In this setting, the decision making takes place inside the source encoder, and the set of decision variables is $\mathscr{D}=\left\{\Delta t_{1: M}, \Delta R_{1: M}, \Delta q_{1: M}\right\}$ where $\Delta t_{1: M}$ with $\Delta t_{s}=$ $t_{s}-t_{s-1}$ and $t_{0}=0$ is the set of time intervals, $\Delta R_{1: M}$ is the set of mode parameters, and $\Delta q_{1: M}$ is the set of quantization step-sizes.

\section{B. Fisher Information Matrix Dynamics}

The conditional distribution $p\left(x_{t} \mid z_{1: s}\right)=N\left(\hat{x}_{t}, P_{t}\right)$, which is Gaussian given the system's model introduced before, evolves in time due to the system dynamics, and is updated at time instants $t_{s}$ due to the measurements.

Consider the transformation $I_{t}=P_{t}^{-1}$ and $\dot{I}_{t}=-I_{t} \dot{P}_{t} I_{t}$ where $I_{t}$ is the Fisher information matrix (FIM) [16]. Following the Kolmogorov forward equation [14], which in our case reduces to the propagation of the conditional mean and conditional covariance matrix of the state, the rate of change 
of the FIM during the interval $\left[t_{s-1}, t_{s}\right)$, in which there are no measurements, is obtained as

$$
\dot{I}_{t}=-A_{t}^{T} I_{t}-I_{t} A_{t}-I_{t} B_{t} Q_{t} B_{t}^{T} I_{t}, \quad t \in\left[t_{s-1}, t_{s}\right)
$$

with initial condition $I_{s-1}=I_{(s-1)^{-}}+\Delta I_{s-1}$ where we mean by $I_{s^{-}}$the left hand side limit of $I_{t}$ as $t$ tends to $t_{s}$ from the left.

In addition, following the Bayes' rule [14], the change in the FIM at the time instant $t_{s}$, when a measurement is available, is obtained as

$$
\Delta I_{s}=I_{s}-I_{s^{-}}=C_{s}^{T} \bar{R}_{s}^{-1} C_{s} \triangleq G_{s}
$$

where

$$
\bar{R}_{s}=R+\Delta R_{s}+\frac{\Delta q_{s}^{2}}{12} I_{p}
$$

and $G_{s}$ is called quality matrix.

Remark 4: The time evolution of the FIM in the continuous-discrete time iterative filtering problem is characterized by the jump dynamics defined by the equations (4), (5). Hence, given the decision set $\mathscr{D}$ and the initial FIM the whole trajectory of the FIM can be obtained.

\section{Measure of Information}

Let the agent's knowledge regarding the state of the process given the measurements at times $t_{s}, t_{s} \leq t$, be represented by the conditional distribution $p\left(x_{t} \mid z_{1: s}\right)=$ $N\left(\hat{x}_{t}, P_{t}\right)$. The differential entropy [16] of the knowledge is given by

$$
H\left(x_{t} \mid z_{1: s}\right)=\frac{1}{2} \log \left[(2 \pi e)^{n} \operatorname{det} I_{t}^{-1}\right]
$$

where $n$ is the dimension of $x_{t}$.

Definition 1 (Information from agent's perspective):

Information is the change in the knowledge possessed by the agent about the state of the process given the transmitted measurements over the time interval $\left[t_{0}, t\right]$ with $t \geq t_{s}$, i.e.,

$$
\Pi_{t} \triangleq H_{0}-H\left(x_{t} \mid z_{1: s}\right)
$$

where $H_{0}$ is the entropy at the reference time $t_{0}$.

In fact, this information has a destination (i.e., the agent) and is defined with respect to an epoch (i.e., time $t_{0}$ ).

Remark 5: Stochastic dynamical systems evolve in the direction in which entropy increases. The dissipated information is the amount of information loss through the natural evolution of the system. However, the entropy can decrease as a result of measurements. The mutual information between the state and a measurement is the amount of information gain about the state provided by that measurement.

For maximizing the cumulative information about the state of the process, from the measurements, which is the integral of the information defined in (8) over the time horizon $[0, T]$, we can minimize the following cost function:

$$
J_{\mathrm{CT}}\left(I_{0}, \mathscr{D}\right)=\int_{0}^{T} H\left(x_{t} \mid z_{1: s}\right) d t
$$

where $I_{0}$ is the initial FIM.

\section{Energy Constraint Set}

We consider that the observer has a constraint on the energy resource for the directed communication to the agent. Let $e$ be the total amount of energy available to the observer over the time horizon $[0, T]$. We define the energy constraint set $\mathscr{C}(e)$ as a function of the total energy as follows

$$
\mathscr{C}(e)=\left\{\mathscr{D} \mid \sum_{s=1}^{M} \rho\left(\Delta R_{s}, \Delta q_{s}\right) \leq e\right\}
$$

where $\rho\left(\Delta R_{s}, \Delta q_{s}\right)$ is the energy consumed per measurement and transmission.

Remark 6: Given that $\Delta R_{s}$ and $\Delta q_{s}$ are selected from finite sets, $\rho\left(\Delta R_{s}, \Delta q_{s}\right)$ associated to each setting can be evaluated.

The constraint set confines the set of admissible decision variables. The properties of the constraint set are:

i) $\mathscr{C}\left(e_{1}\right) \subseteq \mathscr{C}\left(e_{2}\right)$ if $e_{1} \leq e_{2}$,

ii) $\mathscr{D} \in \mathscr{C}(0)$ implies $M=0$,

iii) There exists $\mathscr{D} \in \mathscr{C}(\infty)$ such that $\Delta t_{s} \rightarrow 0, \Delta q_{s} \rightarrow 0$, and $\Delta R_{s}$ corresponds to the highest precision mode for all $s=1,2, \ldots, M$,

where (iii) implies that the observer can transmit its measurements continually with the highest rate and quality.

\section{E. Objective}

Now, we are able to express the main problem of this paper.

Problem 1: Given the system defined by the equations (4), (5), design a source encoder parametrized by $\mathscr{D}$ that maximizes the integral of the information defined in (8) subject to the bound $e$ on the total energy over the time horizon $[0, T]$.

Equivalently,

$$
\begin{array}{ll}
\text { minimize } & J_{\mathrm{CT}}\left(I_{0}, \mathscr{D}\right) \\
\text { subject to } & \mathscr{D} \in \mathscr{C}(e),
\end{array}
$$

given the FIM dynamics.

\section{MAXIMIZATION OF INFORMATION}

We discretize the time variable with $t_{k}=k \epsilon$ and $N=$ $T / \epsilon$ where $\epsilon$ is time discretization step-size. Let $\Delta R_{s}$ and $\Delta q_{s}$ take values in finite sets with cardinalities $L_{1}$ and $L_{2}$ respectively, then the quality matrix $G_{s}$ takes values in a finite set with cardinality $L=L_{1} L_{2}$; i.e., we have $G_{k} \in$ $\left\{G^{1}, \ldots, G^{L}\right\}$. We define a logical variable $\delta_{k}^{i}$ as

$$
\delta_{k}^{i}= \begin{cases}1, & \text { if } t_{k}=t_{s} \text { and } G_{k}=G^{i}, \\ 0, & \text { otherwise. }\end{cases}
$$

Notice that by specifying $\delta=\left\{\delta_{1}^{1}, \ldots, \delta_{N}^{L}\right\}$ the decision set $\mathscr{D}$ is determined.

Using the Euler method and the equation (12), we write the FIM dynamics by the following difference equation

$$
\begin{aligned}
I_{k}= & -\epsilon A_{k-1}^{T} I_{k-1}-\epsilon I_{k-1} A_{k-1}+I_{k-1} \\
& -\epsilon I_{k-1} B_{k-1} Q_{k-1} B_{k-1}^{T} I_{k-1}+\sum_{i=1}^{L} G^{i} \delta_{k}^{i} .
\end{aligned}
$$


Furthermore, following equation (12) the energy constraint set is described by

$$
\sum_{k=1}^{N} \sum_{i=1}^{L} e^{i} \delta_{k}^{i} \leq e
$$

where $e^{i}$ is the energy level associated with $G^{i}$. To guarantee unique selection of a precision mode and of a quantization step-size, the following condition is necessary

$$
\sum_{i=1}^{L} \delta_{k}^{i} \leq 1
$$

Moreover, from equations (7), (9), the cost function in discrete time is defined as

$$
J_{\mathrm{DT}}\left(I_{0}, \mathscr{D}\right)=\frac{\epsilon}{2} \sum_{k=0}^{N}\left[n \log _{2} 2 \pi e-\log _{2} \operatorname{det} I_{k}\right] .
$$

Then, the optimal decision set $\delta^{*}$ is obtained by solving the following finite horizon mixed-integer nonlinear optimization problem:

$$
\begin{array}{ll}
\operatorname{minimize} & \psi_{1}\left(I_{0}, \delta\right)=-\sum_{k=0}^{N} \log _{2} \operatorname{det} I_{k} \\
\text { subject to } \quad & I_{k}=-\epsilon A_{k-1}^{T} I_{k-1}-\epsilon I_{k-1} A_{k-1}+I_{k-1} \\
& -\epsilon I_{k-1} B_{k-1} Q_{k-1} B_{k-1}^{T} I_{k-1}+\sum_{i=1}^{L} G^{i} \delta_{k}^{i}, \\
& \sum_{k=1}^{N} \sum_{i=1}^{L} e^{i} \delta_{k}^{i} \leq e, \quad \sum_{i=1}^{L} \delta_{k}^{i} \leq 1,
\end{array}
$$

with variables $I_{k} \succ 0$ and $\delta_{k}^{i} \in\{0,1\}$ for all $k=$ $1,2, \ldots, N$, and with initial condition $I_{0}$. Notice that the cost function $\psi_{1}\left(I_{0}, \delta\right)$ incorporates the non-constant parts of the cost function in (16).

Proposition 1: The optimization problem in (17) is feasible, and the global minimum is achievable.

Proof: $\delta_{k}^{i}=0$ for all $i$ and $k$ is an admissible solution for which the cost function in (17) is finite and the problem is feasible. The parameter set of all allowed solutions is finite, and therefore there exists a global minimum.

Proposition 2: Let $J_{\mathrm{DT}}\left(I_{0}, \mathscr{D}^{*}\right)$ be the optimal value of the cost function in (16) corresponding to the solution of the discrete-time optimization problem in (17) and $J_{\mathrm{CT}}\left(I_{0}, \mathscr{D}^{*}\right)$ be the optimal value of the continuous-time optimization problem in (11). Then, for any $\gamma>0$ there exists time discretization step-size $\epsilon>0$ such that

$$
\left|J_{\mathrm{CT}}\left(I_{0}, \mathscr{D}^{*}\right)-J_{\mathrm{DT}}\left(I_{0}, \mathscr{D}^{*}\right)\right| \leq \gamma .
$$

Proof: The error in the (instantaneous) FIM with respect to the reference case (when $\epsilon \rightarrow 0$ ) decreases when $\epsilon$ decreases. This implies that the error between the costs $J_{\mathrm{DT}}\left(I_{0}, \mathscr{D}^{*}\right)$ and $J_{\mathrm{CT}}\left(I_{0}, \mathscr{D}^{*}\right)$ also decreases. Hence, we can find $\epsilon$ such that the error between the costs is less than $\gamma$.

In order to compute the optimal solution of the optimization problem in (17), one can use the branch and bound (BB) algorithm [17]. In the BB algorithm (described in
Algorithm 1), a queue (initially contains the feasible set) is iteratively divided into disjoint subsets. In each iteration, a subset is selected, and a lower bound and an upper bound (the cost of a feasible solution) of the subset are calculated. A subset is pruned from the queue if its lower bound is worse than the best achieved upper bound, if its subproblem is infeasible, or if its lower bound is equal to its upper bound. Otherwise, it is branched into children. The algorithm stops when the queue is empty or the gap between the best achieved upper and lower bounds is less than a specific tolerance $\epsilon_{0}$.

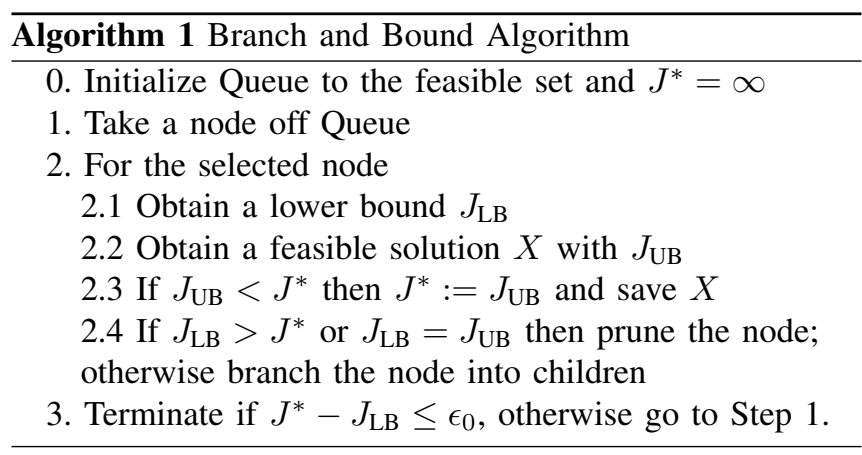

However, the BB algorithm is computationally expensive. In the next section, we develop an efficient algorithm for obtaining a suboptimal solution of the optimization problem in (17). The bounds obtained in the next section can also be used in Algorithm 1.

\section{BOUNDS ON THE INFORMATION AND A SUBOPTIMAL SDP-BASED SOLUTION}

We utilize an SDP relaxation [18] to obtain a lower bound on the optimal value of the optimization problem in (17). In addition, based on this SDP we find a suboptimal admissible solution.

Theorem 1: A lower bound on the optimal value of the optimization problem in (17) is given by the following SDP:

$$
\begin{array}{ll}
\operatorname{minimize} & \psi_{2}\left(I_{0}, \delta\right)=-\sum_{k=0}^{N} \log _{2} \operatorname{det} I_{k} \\
\text { subject to } \quad & I_{k}=-\epsilon A_{k-1}^{T} I_{k-1}-\epsilon I_{k-1} A_{k-1}+I_{k-1} \\
& -\epsilon D_{k-1}+\sum_{i=1}^{L} G^{i} \delta_{k}^{i}, \\
& {\left[\begin{array}{l}
D_{k} \quad I_{k} \\
I_{k} \quad\left(B_{k} Q_{k} B_{k}^{T}\right)^{-1}
\end{array}\right] \succeq 0,} \\
& \sum_{k=1}^{N} \sum_{i=1}^{L} e^{i} \delta_{k}^{i} \leq e, \quad \sum_{i=1}^{L} \delta_{k}^{i} \leq 1
\end{array}
$$

with variables $I_{k} \succ 0, D_{k} \succ 0$, and $\delta_{k}^{i} \in[0,1]$ for all $k=1,2, \ldots, N$, and with initial conditions $I_{0}$ and $D_{0}=$ $I_{0} B_{0} Q_{0} B_{0}^{T} I_{0}$.

Proof: Fist note that the function $-\log _{2} \operatorname{det} I_{k}$ is convex in $I_{k}$. Following the optimization problem in (17), we relax the integrality constraint to $\delta_{k}^{i} \in[0,1]$. Then, we aim at 
relaxing the quadratic term in the FIM dynamics. Since this equality constraint does not influence the set of admissible decisions $\delta_{k}^{i}$, such a relaxation does not violate the feasibility of the problem. By defining $D_{k}=I_{k} B_{k} Q_{k} B_{k}^{T} I_{k}$, we have

$$
\begin{aligned}
I_{k}= & -\epsilon A_{k-1}^{T} I_{k-1}-\epsilon I_{k-1} A_{k-1}+I_{k-1} \\
& -\epsilon D_{k-1}+\sum_{i=1}^{L} G^{i} \delta_{k}^{i}
\end{aligned}
$$

which is a linear equation. We substitute the new constraint $D_{k}=I_{k} B_{k} Q_{k} B_{k}^{T} I_{k}$ with its convex relaxation, i.e., $D_{k}-$ $I_{k} B_{k} Q_{k} B_{k}^{T} I_{k} \succeq 0$. This nonlinear convex inequality can be converted into an LMI by using the Schur complement:

$$
\left[\begin{array}{cc}
D_{k} & I_{k} \\
I_{k} & \left(B_{k} Q_{k} B_{k}^{T}\right)^{-1}
\end{array}\right] \succeq 0 .
$$

Substituting the constraints (13) and $\delta_{k}^{i} \in\{0,1\}$ with the constraints (20), (21), and $\delta_{k}^{i} \in[0,1]$ in the optimization problem in (17), and following the convexity of the cost function we obtain the relaxed problem (19) which yields a lower bound for the original problem.

We can use the lower bound provided by Theorem 1 in an approximation algorithm (described in Algorithm 2) to obtain a suboptimal admissible solution.

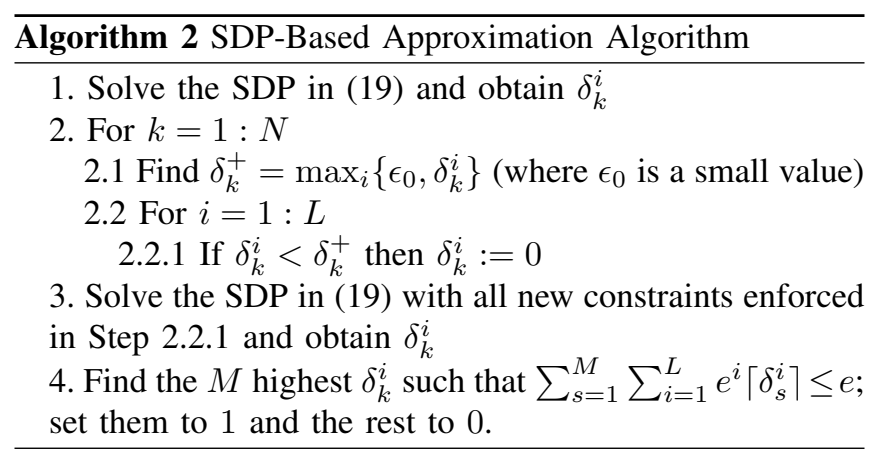

The cost corresponding to the solution given by Algorithm 2 is an upper bound on the optimal value of the optimization problem in (17).

\section{A. Information Envelope}

We can obtain lower and upper bounds on the FIM if there is no constraint on the energy expenditure. A lower bound on the FIM is given by

$$
\begin{aligned}
V_{k} & =-\epsilon A_{k-1}^{T} V_{k-1}-\epsilon V_{k-1} A_{k-1}+V_{k-1} \\
& -\epsilon V_{k-1} B_{k-1} Q_{k-1} B_{k-1}^{T} V_{k-1}
\end{aligned}
$$

and an upper bound on the FIM is obtained by

$$
\begin{array}{r}
S_{k}=-\epsilon A_{k-1}^{T} S_{k-1}-\epsilon S_{k-1} A_{k-1}+S_{k-1} \\
-\epsilon S_{k-1} B_{k-1} Q_{k-1} B_{k-1}^{T} S_{k-1}+\sum_{i=1}^{L} G^{i}
\end{array}
$$

with initial conditions $V_{0}=S_{0}=I_{0}$.

We define the information envelope associated with the system as a region with boundaries specified by the information corresponding to $V_{k}$ and $S_{k}$.

\section{An OPtIMIZED LMI-BASED EVENT-TRIGGERING MECHANISM}

In this section, we assume that $G_{s}=G^{0}$ with the associated energy level $e^{0}$ is fixed. We propose an eventtriggering mechanism based on an LMI which yields eventdriven sampling. The LMI-based event-triggering mechanism that we are interested in is described by

$$
\delta_{k}= \begin{cases}0, & \text { if } I_{k} \succeq \Theta, \\ 1, & \text { otherwise },\end{cases}
$$

where $\Theta$ is a threshold on the FIM. In the following, we optimize this threshold by exploiting the optimization problem in (17).

The inequality in (24) can be converted into the following two constraints:

$$
\begin{aligned}
& \lambda_{\max }\left(\Theta-I_{k}\right)\left(1-\delta_{k}\right) \leq 0, \\
& \lambda_{\max }\left(\Theta-I_{k}\right) \delta_{k} \geq 0,
\end{aligned}
$$

where $\lambda_{\max }\left(\Theta-I_{k}\right)$ is the maximum eigenvalue of the matrix $\Theta-I_{k}$. Adding the constraints (25), (26) to the optimization problem in (17), we have

$$
\begin{array}{ll}
\operatorname{minimize} & \psi_{3}\left(I_{0}, \Theta\right)=-\sum_{k=0}^{N} \log _{2} \operatorname{det} I_{k} \\
\text { subject to } & I_{k}=-\epsilon A_{k-1}^{T} I_{k-1}-\epsilon I_{k-1} A_{k-1}+I_{k-1} \\
& -\epsilon I_{k-1} B_{k-1} Q_{k-1} B_{k-1}^{T} I_{k-1}+G^{0} \delta_{k} \\
& \lambda_{\max }\left(\Theta-I_{k}\right)\left(1-\delta_{k}\right) \leq 0 \\
& \lambda_{\max }\left(\Theta-I_{k}\right) \delta_{k} \geq 0, \quad \sum_{k=1}^{N} e^{0} \delta_{k} \leq e
\end{array}
$$

with variables $I_{k} \succ 0, \Theta \succ 0$, and $\delta_{k} \in\{0,1\}$ for all $k=1,2, \ldots, N$, and with initial condition $I_{0}$. The optimal threshold $\Theta^{*}$ of the event-triggering mechanism in (24) is obtained by solving this optimization problem.

Proposition 3: Assume that $G_{s}=G^{0}$ is fixed. The optimal value of the optimization problem in (27) is greater than or equal to that of the optimization problem in (17), i.e.,

$$
\psi_{1}\left(I_{0}, \delta^{*}\right) \leq \psi_{3}\left(I_{0}, \Theta^{*}\right) .
$$

Proof: Adding the new constraints (25) and (26) reduces the size of the feasible set of the optimization problem in (17). Therefore, $\psi_{1}\left(I_{0}, \delta^{*}\right) \leq \psi_{3}\left(I_{0}, \Theta^{*}\right)$.

\section{An Illustrative EXAmPle}

In this section, we present numerical and simulation results for simple stable and unstable systems.

Example 1: Consider a stable and an unstable system with dynamics defined by the equations (1), (2), (3) with $A_{t}=-0.1,0.1$ for the stable and the unstable systems respectively, $B_{t}=1, C_{s}=\sqrt{0.1}, Q_{t}=0.5$, and $P_{0}=2$. The discretization step-size is $\epsilon=0.1$, the horizon is $N=40$, the total energy is $e=42$, and the cost is $\psi=-\sum_{k=0}^{N} \log _{2} I_{k}$.

The quality matrices are $G^{1}=0.08, G^{2}=0.09$ (which specify quantization step-sizes and precision parameters) with energy levels $e^{1}=5, e^{2}=6$. We used the $\mathrm{BB}$, the 


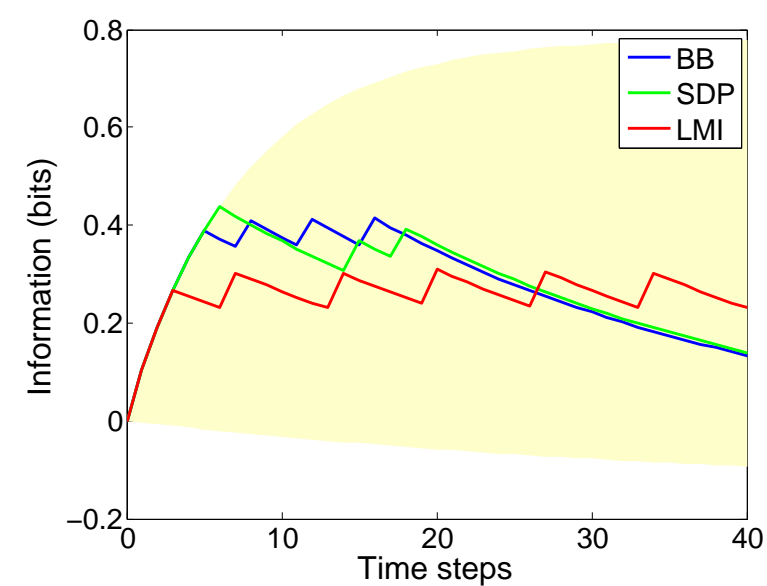

Fig. 2. Information diagrams for the stable system with the associated costs $\psi_{\mathrm{BB}}=18.12, \psi_{\mathrm{SDP}}=18.17$, and $\psi_{\mathrm{LMI}}=20.19$. The shaded area represents the information envelope.

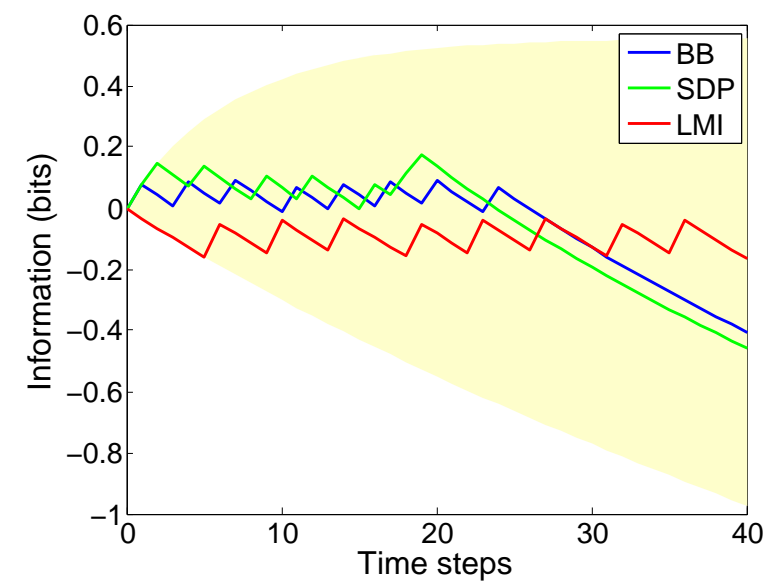

Fig. 3. Information diagrams for the unstable system with the associated costs $\psi_{\mathrm{BB}}=45.24, \psi_{\mathrm{SDP}}=45.50$, and $\psi_{\mathrm{LMI}}=48.71$. The shaded area represents the information envelope.

SDP, and the approximation algorithms introduced in this paper to obtain the maximum information and its lower and upper bounds. For the stable system, the optimal cost and its lower and upper bounds are $\psi_{\mathrm{BB}}=17.55, \psi_{\mathrm{LB}}=17.28$, and $\psi_{\mathrm{UB}}=18.22$, respectively; and for the unstable system they are $\psi_{\mathrm{BB}}=44.51, \psi_{\mathrm{LB}}=44.01$, and $\psi_{\mathrm{UB}}=45.33$, respectively. Furthermore, we compare the optimal sampling obtained by the BB algorithm with the SDP-based and the LMI-based samplings for $G^{0}=0.08$. Notice that in this example the LMI reduces to a linear inequality. Fig. 2 and 3 show the information diagrams with the optimal, the SDPbased, and the LMI-based samplings for the stable and the unstable systems, respectively. The associated costs for the stable system are $\psi_{\mathrm{BB}}=18.12, \psi_{\mathrm{SDP}}=18.17$, and $\psi_{\mathrm{LMI}}=$ 20.19 (with $\Theta^{*}=0.70$ ); and for the unstable system they are $\psi_{\mathrm{BB}}=45.24, \psi_{\mathrm{SDP}}=45.50$, and $\psi_{\mathrm{LMI}}=48.71$ (with $\left.\Theta^{*}=0.41\right)$. It is observed that the optimized LMI-based event-driven sampling is worse than the optimal sampling.

\section{CONCLUSION}

In this paper, we studied the maximum information that can be transferred from an observer to an agent over a finite horizon while there is a limitation on the energy resource of the observer for the directed communication to the agent. We formulated the maximization of information problem, and developed an efficient algorithm for obtaining bounds on the optimal value and a suboptimal solution of this problem. We proposed an optimized event-triggering mechanism based on an LMI, and showed that the performance of the LMIbased event-driven sampling is inferior to that of the optimal sampling.

\section{ACKNOWLEDGMENT}

This work has been carried out with the support of the Technische Universität München - Institute for Advanced Study, funded by the German Excellence Initiative. John S. Baras' research was also supported by NSF grant CNS1035655, and by DARPA (ARO) grant W911NF1410384.

\section{REFERENCES}

[1] L. Meier, J. Peschon, and R. M. Dressler, "Optimal control of measurement subsystems," IEEE Trans. Automatic Control, vol. 12, no. 5, pp. 528-536, 1967.

[2] H. J. Kushner, "On the optimum timing of observations for linear control systems with unknown initial state," IEEE Trans. Automatic Control, vol. 9, no. 2, pp. 144-150, 1964.

[3] M. Rabi, G. V. Moustakides, and J. S. Baras, "Adaptive sampling for linear state estimation," SIAM Journal on Control and Optimization, vol. 50, no. 2, pp. 672-702, 2012.

[4] A. Molin and S. Hirche, "An iterative algorithm for optimal eventtriggered estimation," in Proc. of the IFAC Conf. on Analysis and Design of Hybrid Systems, pp. 64-69, 2012.

[5] J. Sijs and M. Lazar, "Event based state estimation with time synchronous updates," IEEE Trans. Automatic Control, vol. 57, no. 10, pp. 2650-2655, 2012.

[6] S. Trimpe and R. D'Andrea, "Event-based state estimation with variance-based triggering," IEEE Trans. Automatic Control, vol. 59, no. 12 , pp. 3266-3281, 2014.

[7] J. S. Baras and A. Bensoussan, "Optimal sensor scheduling in nonlinear filtering of diffusion processes," SIAM Journal on Control and Optimization, vol. 27, no. 4, pp. 786-813, 1989.

[8] A. V. Savkin, R. J. Evans, and E. Skafidas, "The problem of optimal robust sensor scheduling," Systems \& Control Letters, vol. 43, no. 2, pp. 149-157, 2001.

[9] V. Krishnamurthy and R. J. Evans, "Hidden Markov model multiarm bandits: a methodology for beam scheduling in multitarget tracking," IEEE Trans. Signal Process., vol. 49, no. 12, pp. 2893-2908, 2001.

[10] V. Gupta, T. H. Chung, B. Hassibi, and R. M. Murray, "On a stochastic sensor selection algorithm with applications in sensor scheduling and sensor coverage," Automatica, vol. 42, no. 2, pp. 251-260, 2006.

[11] H. Sandberg, M. Rabi, M. Skoglund, and K. H. Johansson, "Estimation over heterogeneous sensor networks," in Proc. of the IEEE Conf. on Decision and Control, pp. 4898-4903, 2008.

[12] W. Wu and A. Arapostathis, "Optimal sensor querying: General Markovian and LQG models with controlled observations," IEEE Trans. Automatic Control, vol. 53, no. 6, pp. 1392-1405, 2008.

[13] J. L. Ny, E. Feron, and M. A. Dahleh, "Scheduling continuoustime kalman filters," IEEE Trans. Automatic Control, vol. 56, no. 6, pp. 1381-1394, 2011.

[14] A. Jazwinski, "Filtering for nonlinear dynamical systems," IEEE Trans. Automatic Control, vol. 11, no. 4, pp. 765-766, 1966.

[15] D. Marco and D. L. Neuhoff, "The validity of the additive noise model for uniform scalar quantizers," IEEE Trans. Information Theory, vol. 51, no. 5, pp. 1739-1755, 2005.

[16] D. Guo, S. Shamai, and S. Verdú, "The interplay between information and estimation measures," Foundations and Trends in Signal Processing, vol. 6, no. 4, pp. 243-429, 2012.

[17] D. P. Bertsekas, Nonlinear programming. Athena Scientific, 1999.

[18] S. Boyd and L. Vandenberghe, "Semidefinite programming relaxations of non-convex problems in control and combinatorial optimization," in Communications, Computation, Control, and Signal Processing, pp. 279-287, Springer, 1997. 